\title{
Limited Comp Handover Algorithm For LTE-Advanced
}

\author{
Cheng-Chung Lin, Kumbesan Sandrasegaran, Xinning Zhu, and Zhuliang Xu
}

Faculty of Engineering and Information Technology, University of Technology, Sydney, NSW, Australia

Correspondence should be addressed to Cheng-Chung Lin; cheng-chung.lin@eng.uts.edu.au

Received 31 August 2012; Revised 17 December 2012; Accepted 11 January 2013

Academic Editor: Stavros Koubias

Copyright (C) 2013 Cheng-Chung Lin et al. This is an open access article distributed under the Creative Commons Attribution License, which permits unrestricted use, distribution, and reproduction in any medium, provided the original work is properly cited.

Coordinated multipoint (CoMP) transmission and reception is the key technique in LTE-Advanced to improve the cell-edge throughput and/or system throughput. Joint processing (JP) in CoMP technology provides multiple data transmission points for each user among multiple cooperated radio base stations. Hard handover mechanism is adopted to be used in LTE-Advanced. Standard hard handover algorithm could not satisfy the concept of JP in CoMP in LTE-A due to the constraint of single connection for each user at any time. While the radio resources in the system are fixed, the more multiple data connections a user has, the more radio resources are used for the extra data connections, thus the lower capacity a system becomes. Therefore a new handover algorithm that not only supports JP in CoMP but also takes system capacity into consideration in LTE-A system is necessary. This paper proposes a new handover algorithm known as Limited CoMP Handover Algorithm to support JP in CoMP and overcome the system capacity issue. System performance of Limited CoMP Handover Algorithm is evaluated and compared with open literature handover algorithm via simulation in this paper. The simulation results show that Limited CoMP Handover Algorithm outperforms open literature handover algorithm by having shorter system delay and less system load whilst maintaining a higher system throughput in a high congested network.

\section{Introduction}

Long-Term Evolution-Advanced (also known as LTEAdvanced, LTE-A, or LTE Release 10) is a mobile communication standard proposed by 3rd Generation Partnership Project (3GPP) in September 2009 as a major enhancement of the LTE standard. LTE-A was accepted as a candidate $4 \mathrm{G}$ system to improve LTE system to meet the international mobile telecommunications advanced (IMTAdvanced) requirements [1] issued by the international telecommunication union-radiocommunication sector (ITU-R). LTE-A is a purely packet switched radio access technology that supports higher capacity, coverage, and data rates (i.e., up to $1 \mathrm{Gbps}$ in downlink and up to $500 \mathrm{Mbps}$ in uplink) [2] than earlier wireless systems. LTE-A uses Orthogonal Frequency Division Multiple Access (OFDMA), which is a variant of OFDM (Orthogonal Frequency Division Multiplexing), as its access technology in the downlink, while Single-Carrier Frequency Division Multiple Access (SCFDMA) is used in the uplink multiple access scheme [3]. OFDMA is a multicarrier access technology that divides the wide available bandwidth into multiple equally spaced and mutually orthogonal subcarriers. The smallest transmission unit in the downlink LTE-A system is known as a pair of resource blocks $(\mathrm{RB})$ that contains 12 subcarriers $(180 \mathrm{kHz}$ total bandwidth) over a slot duration of $0.5 \mathrm{~ms}$ [4].

The radio architecture of LTE-A is shown in Figure 1. The network architecture consists of three elements and two interfaces: evolved-NodeB (eNodeB), Mobile Management Entity (MME), and Serving Gateway (S-GW)/Packet Data Network Gateway (P-GW) and S1 and X2 interface, respectively [5].

There are a number of key features introduced in LTEA, including carrier aggregation, downlink and uplink spatial multiplexing enhancement, coordinated multipoint (CoMP) transmission and reception, relaying nodes, and heterogeneous networks compatibility [6]. Carrier aggregation permits an eNodeB to group several distinct channels into one logical channel. This results in high peak data rate of $1 \mathrm{Gbps}$ in downlink and $500 \mathrm{Mbps}$ in uplink can be achieved with bandwidth extension from $20 \mathrm{MHz}$ to $100 \mathrm{MHz}$ in LTEAdvanced. Downlink spatial multiplexing using up to eightlayer multiple-input multiple-output (MIMO) and uplink 


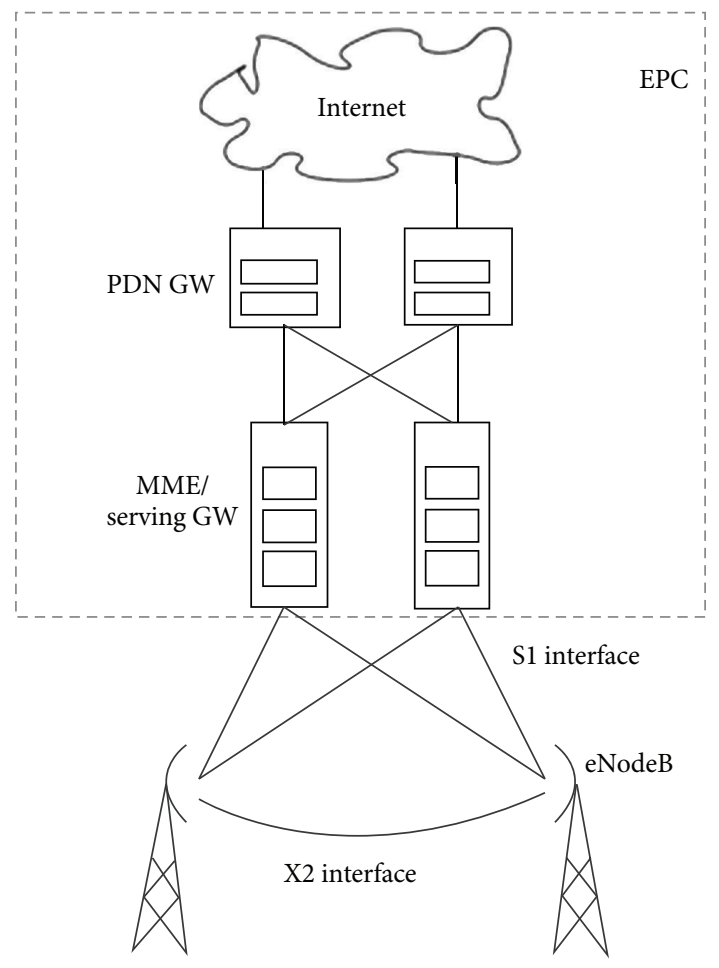

FIGURE 1: Network architecture of LTE-A [5].

spatial multiplexing using four-layer MIMO increase average and peak data rate and the cell edge throughput [7]. Both Relaying nodes and heterogeneous networks compatibility provide coverage and capacity in areas difficult or expensive to reach using the traditional approach.

CoMP transmission and reception is the key technique in LTE-A to improve the cell-edge throughput and/or system throughput. Two types of CoMP schemes were evaluated for LTE-A system: Joint Processing (JP) and Coordinated Scheduling/Beamforming (CS/CB). JP provides multiple data transmissions for each user equipment (UE) among multiple cooperated eNodeBs while CS/CB only supports single data transmission for each UE at serving eNodeB with user scheduling/beamforming decisions made with coordination among cooperated eNodeBs [8].

Handover is a mechanism that transfers an on-going call or data session from one base station (BS) to another BS (hard handover) or one sector to another sector within the same BS (soft(er) handover). Hard handover refers a UE starts handover to the target cell by breaking the current existing connection with the source cell before having a new connection with the target cell. Unlike hard handover, soft(er) handover makes the possibility for a UE simultaneously to connect to two or more cells (or cell sectors) during a call or data session. Handover in LTE-A is a hard handover [9]. A single connection for each UE connected with the source eNodeB at any time instant is restricted by the nature of hard handover mechanism. Therefore when the concept of JP in CoMP (allowing multiple data transmissions for each UE at any time instant) comes in LTE-A system, it conflicts the concept of standard hard handover mechanism. A handover mechanism in CoMP is purposed in [10] to solve the conflict. A handover algorithm is needed and used for making a handover decision in handover mechanism. Based on [10], A CoMP Handover Algorithm supporting JP in LTE-A system is implemented and simulated in a previous study [11]. The simulation results have shown that when compared with the standard handover algorithm in LTE system, CoMP Handover Algorithm in LTE-A is able to improve system throughput and minimize packet loss rate (PLR); however this algorithm could lead to system capacity overload and saturated system throughput issues within a high congested network. The reason for the issues is that JP supported UEs in LTE-A acquire multiple radio resources among eNodeBs for their multiple data connections. While the radio resources in the system are fixed, the more multiple data connections a UE has, the more radio resources are used for the extra data connections, thus the lower capacity a system becomes. Hence a new handover algorithm not only supports JP in CoMP but also takes system capacity into consideration in LTE-A system is necessary. A new handover algorithm for LTE-A known as Limited CoMP Handover Algorithm is proposed, evaluated, and compared with CoMP Handover Algorithm in this paper. Limited CoMP Handover Algorithm aims to support JP in CoMP as well as to enhance the capacity and throughput in LTE-A system.

The rest of this paper is organized as follows: Section 2 describes CoMP Handover Algorithm and Limited CoMP Handover Algorithm in detail. The performance metrics used for evaluation and simulation environment are discussed in Sections 3 and 4, respectively. Section 5 contains results of the performance evaluation, and conclusions are summarized in Section 6.

\section{CoMP Handover Algorithms}

CoMP Handover Algorithm and purposed Limited CoMP Handover Algorithm are going to be individually discussed in the following Sections 2.1 and 2.2 followed by a deep discussion about the philosophy of Limited CoMP Handover Algorithm, the differences, and the similarities between these two algorithms in Section 2.3.

2.1. CoMP Handover Algorithm [10]. CoMP Handover Algorithm consists of four elements: serving cell, measurement set, CoMP coordinating set (CCS), and CoMP transmission points (CTP). A serving cell is the cell which takes the responsibility for making handover decision and maintains the connection of each UE to the network. A UE can only attach to one serving cell at each time instant. A measurement set is a set of cells whose RSRPs can be received and reported by the UE and feeds back to the serving cell for making the selection of CCS. A CCS is a set of cells which are selected by the serving cell from the measurement set. Furthermore, A CTP is a set of cells chosen from the CCS by the serving cell for sending downlink data directly to a UE. The size of CCS can be adjusted but the size of CTP can only be less or equal to the size of CCS. Figure 2 demonstrates an example of CoMP in LTE-A system. 


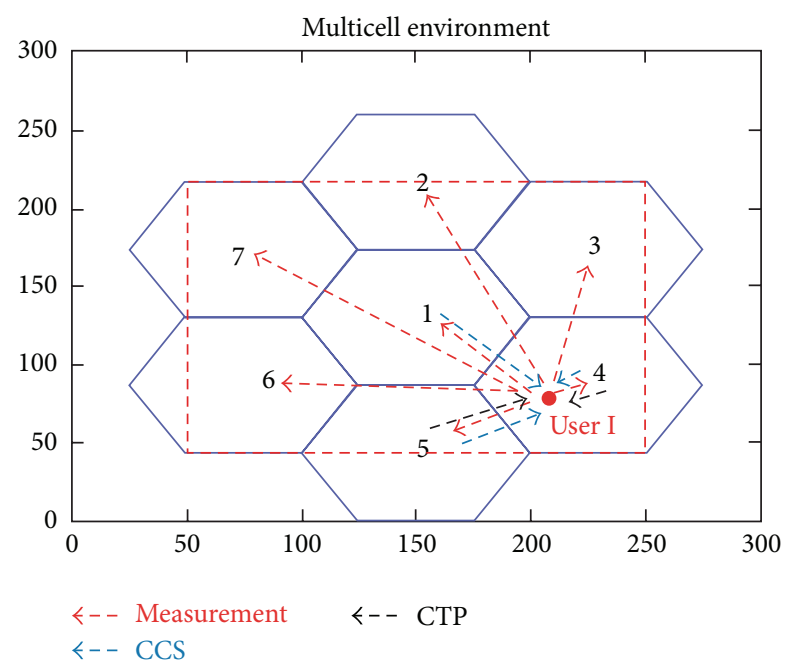

Figure 2: CoMP in LTE-A.

In Figure 2, UE 1's serving cell is assumed as cell 4 and the size of UE 1's CCS and CTP is assumed as 3 and 2, respectively. While UE 1 can measure the signal strength from all cells in Figure 2, a measurement set of cells $\{1,2,3,4,5,6,7\}$ is obtained. A CCS will be a subset of measurement set with a limited size as 3 cells; therefore a set of cells $\{1,4,5\}$ can be obtained as CCS. Moreover, a CTP can be further narrowed down from CCS with a limited size of 2 cells. As a result, a set of cell $\{4,5\}$ is obtained as CTP.

There are three variables involved in CoMP Handover Algorithm: measurement period, handover margin (HOM), and time to trigger (TTT) timer. A measurement period is a time period that is used for checking the handover condition periodically [13]. A handover margin is a constant variable that represents the threshold for the difference in reference signal received power (RSRP) between the serving and the target cells. HOM helps in identifying the most appropriate target cell that a mobile can be handed over to. A TTT value is the time interval that is required for satisfying HOM condition. A combination of TTT and HOM can prevent happening unnecessary handovers, so-called Ping Pong effect [14]. When a mobile is experiencing this effect, it is handed over from a serving cell to a target cell and handed back to original serving cell again in a small period of time [15]. This effect increases the required signalling resources, decreases system throughput, and increases traffic delay caused by buffering the incoming traffic at the target cell when each handover occurs. A handover can only be executed after both of the criteria of TTT and HOM are met. The complete handover decision algorithm is illustrated in Figure 3.

CoMP Handover Algorithm starts when the UE joins the network by camping on the cell whose RSRP is the highest or the cell which was instructed by previous serving cell. Then UE starts to feed back the serving cell with the measurement set which is the RSRP measurements received from all cells in the network. The first measurement period expires immediately due to an update required for the new incoming UE. Serving cell selects a set of cells with highest RSRP in

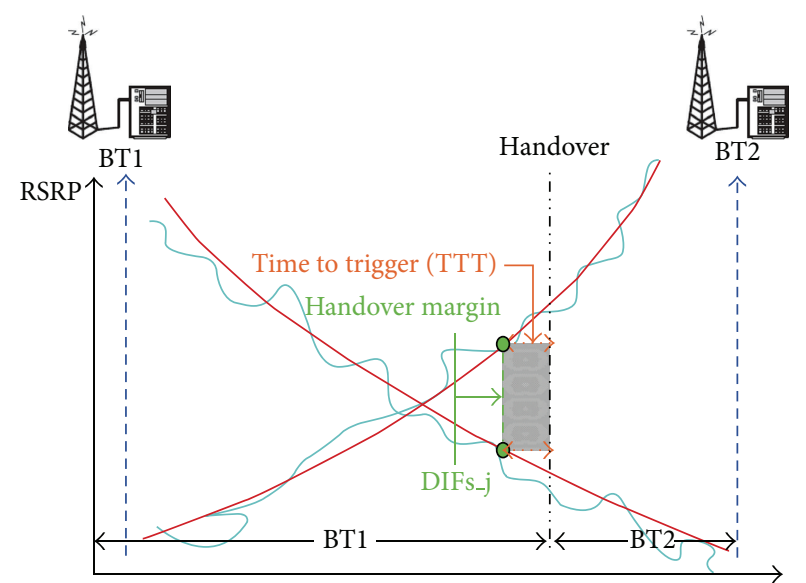

Figure 3: Handover Condition in LTE-A [12].

measurement set as a CCS. Similarly, a set of cells with highest RSRP in CCS will be then selected by the serving cell as the CTP. After the CTP selection is finalized, the serving cell will request all cells in the CTP to start transmitting packets to the UE. When the regular measurement update is required during the transmission, the selection of CCS and CTP for each UE will be repeated by the serving cell to search for updated target cells. A handover is triggered when the condition is satisfied for the TTT time duration expressed as follows:

$$
\mathrm{RSRP}_{T_{-} \mathrm{CTP}}>\mathrm{RSRP}_{S}+\mathrm{HOM},
$$

where $\mathrm{RSRP}_{T_{-} \text {CTP }}$ and $\mathrm{RSRP}_{S}$ are the RSRP received by a UE from the target cells in the CTP and the serving cell, respectively.

Once handover is triggered, the serving cell sends a cancellation message to each of the cell in CTP to cancel the current transmission. A handover command is triggered to instruct the UE to handover to the future serving cell. A complete flowchart of the algorithm is provided in Figure 4.

2.2. Limited CoMP Handover Algorithm. Limited CoMP Handover Algorithm also consists of the same four variables in Section 2.1: serving cell, measurement set, CoMP coordinating set (CCS), and CoMP transmission points (CTP). A serving cell takes the responsibility for making handover decision for each UE in the network. A measurement set is a set of cells whose RSRPs can be received and reported by the $\mathrm{UE}$ and feeds back to the serving cell for making the selection of CCS. A CCS is a subset of the measurement set, and a CTP is a subset of CCS. The same three variables in Section 2.1 are also involved in Limited CoMP Handover Algorithm: measurement period, handover margin (HOM), and time to trigger (TTT) timer. A measurement period acts like a time period that is used for checking the handover condition periodically. A handover margin is a constant variable that represents the threshold for the difference in RSRP between the serving and the target cells. A TTT value is the time interval that is required for satisfying $\mathrm{HOM}$ condition. 


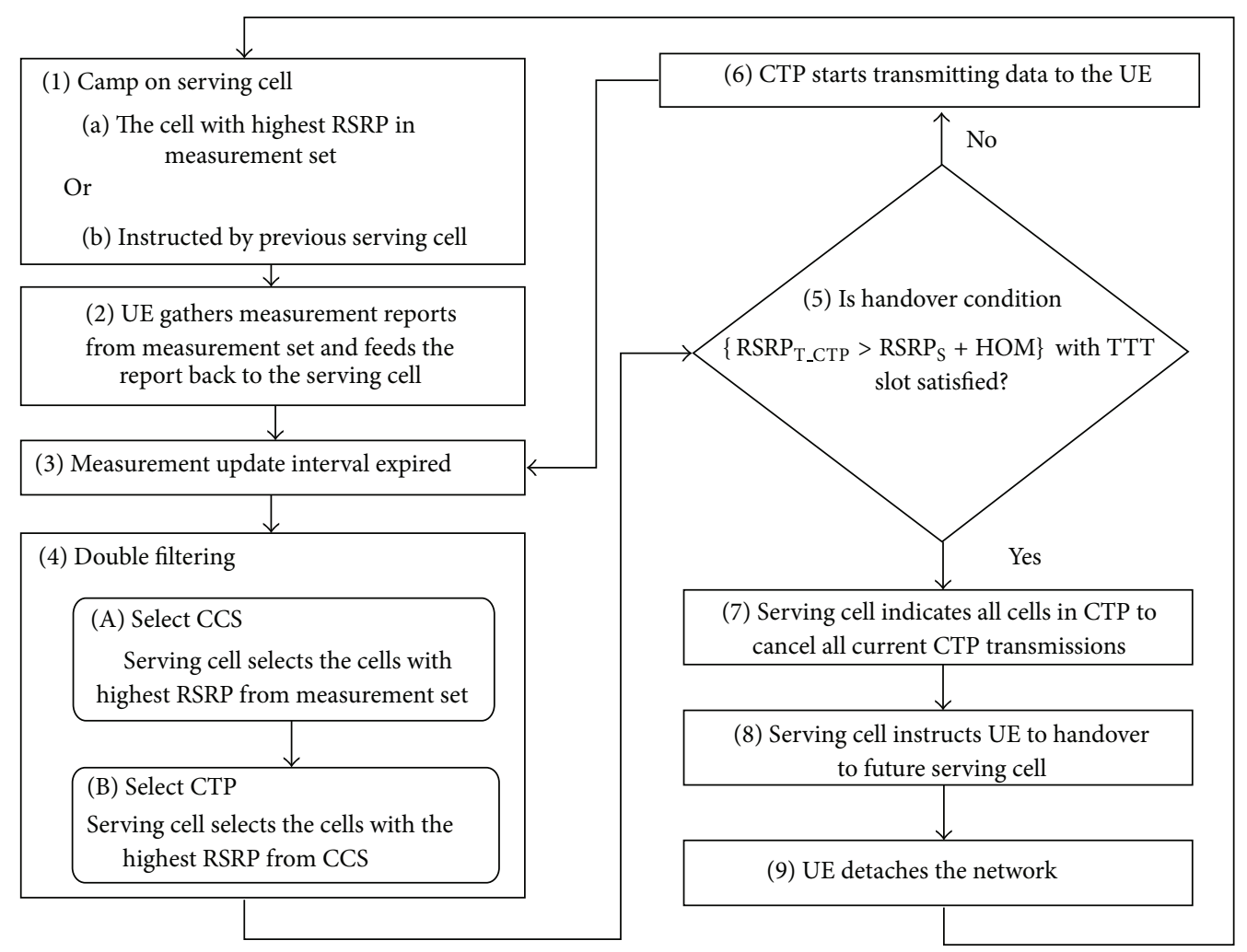

FIgURE 4: Flowchart of CoMP Handover Algorithm in LTE-A.

Limited CoMP Handover Algorithm starts the cell selection/reselection when the UE joins the network by camping on the cell with highest RSRP or the cell which was instructed by a previous serving cell. UE gathers measurement reports which are the RSRP measurements received from the measurement set and feeds the reports back to the serving cell. The first measurement period expires immediately due to an update required for the new incoming UE. Serving cell recursively selects the cell with highest RSRP to be the CCS until reaching the end of measurement set. Moreover, the CTP selection will be recursively executed by the serving cell until reaching the end of CCS based on (2) as follows:

$$
\mathrm{RSRP}_{T_{-} \mathrm{CCS}}<\mathrm{RSRP}_{S}-\mathrm{HOM},
$$

where $\mathrm{RSRP}_{T_{-} \mathrm{CCS}}$ and $\mathrm{RSRP}_{S}$ are the RSRP received by a UE from the target cell in the CCS and the serving cell, respectively.

The target cell in the CCS will be ignored if (2) is satisfied; otherwise the target cell in the CCS will be added into CTP. After CTP selection is finalized, the serving cell performs a handover condition check in CTP based on (3) as follows:

$$
\mathrm{RSRP}_{T_{\text {_CTP }}}>\mathrm{RSRP}_{S}+\mathrm{HOM} \text {, }
$$

where $\mathrm{RSRP}_{T_{-} \text {СTP }}$ and $R S R P_{S}$ are the RSRP received by a UE from the target cell in the CTP and the serving cell, respectively.

A handover is triggered if (3) is satisfied within TTT duration. The serving cell sends out a handover command to instruct the UE to handover to the future serving cell. Lastly, UE detaches from the network and camps on a new serving cell after the interruption time (the time period during which UE disconnects from the network). CTP starts transmitting data to the UE and waits for the next incoming measurement period expired if (2) is not satisfied at any point in time during TTT duration. When the measurement period is not expired, the serving cell directly performs the CTP selection (Step 6 in Figure 5). CTP continues transmitting data to the UE and repeats this process until the next measurement period expires. A complete flowchart of Limited CoMP Handover Algorithm is provided in Figure 5. Note that the red solid line, the blue dash line, and the black long dash dot line in Figure 5 indicate the directions in time domain of measurement period, each other time instant, and both, respectively.

\subsection{Discussion. As described in Section 2.1, CoMP Handover} Algorithm always tracks the highest target cells in measurement set and CCS for the candidate target cells in CTP (Step 4(A) and 4(B) in Figure 4). CoMP Handover Algorithm improves the system throughput by giving multiple connections to each UE at any time instant regardless of their channel condition, but this mechanism also accelerates the current system capacity multiple times as fast reaching its maximum capacity due to the multiple connections needed to be maintained for each UE in the network. Therefore CoMP Handover Algorithm leads to system capacity overload and saturated system throughput issues within a high congested network. Limited CoMP Handover Algorithm comes with 


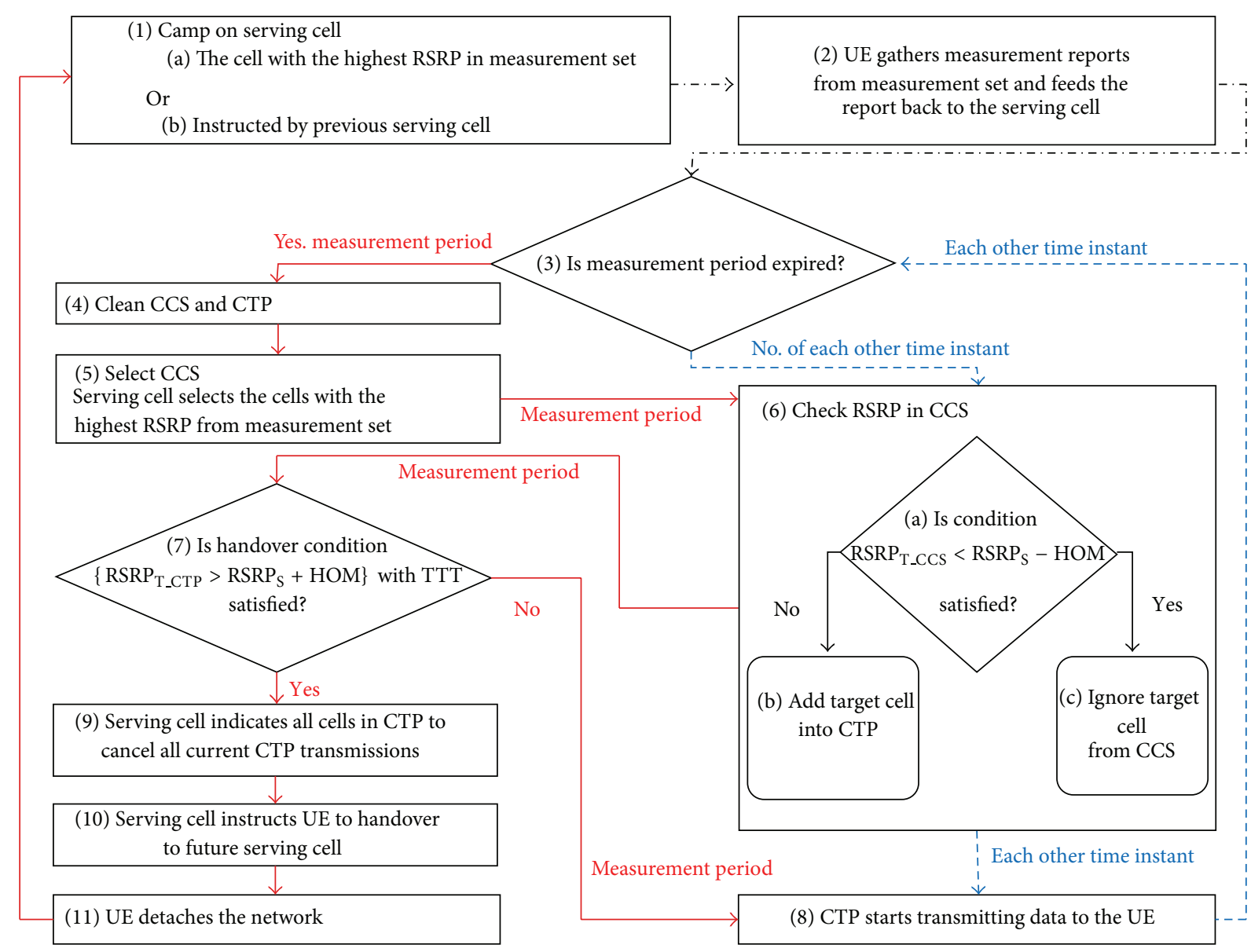

FIGURE 5: Flowchart of Limited CoMP Handover Algorithm in LTE-A.

the concept of having multiple transmissions for each UE when necessary in order to extend the system capacity as high as possible. Limited CoMP Handover Algorithm offers single data transmission for UE with good/fair channel condition (to be more likely, a cell-center UE) in order to save the available radio resources for accommodating more incoming UE while supporting multiple data transmissions when needed for UE with fair/bad channel condition (to be more likely, a cell-edge UE).

The major difference between CoMP Handover Algorithm and Limited CoMP Handover Algorithm is in Step 6 in Figure 5 where Limited CoMP Handover Algorithm tracks the channel quality of each target cell in CTP for each UE at any other time instant by using a window size of HOM (the window size of HOM can be adjusted to adapt to different scenarios) in (2) to identify the channel quality of the target cell compared with the serving cell. If the RSRP of a target cell in CTP which a UE received is significantly lower than the RSRP of the serving cell with a window size of HOM, this UE is categorized as a cell-center UE. Thus the multiple data transmissions for cell-center UEs should be avoided in order to prevent the radio resources overused in other target cells in CTP based on the concept of Limited CoMP Handover Algorithm. On the other hand, if the RSRP of a target cell in CTP which a UE received is within the range of the window size of HOM from the RSRP of the serving cell, this UE is categorized as a cell-edge UE. Thus Limited CoMP Handover Algorithm has to provide multiple data transmissions for celledge UEs to maintain their quality of connections while they are staying in cell-edge areas. This mechanism (Step 6 in Figure 5) helps in eliminating inefficient data transmission at any time instant in the network; therefore Limited CoMP Handover Algorithm is able to maintain the available radio resources more efficiently.

Limited CoMP Handover Algorithm and CoMP Handover Algorithm share most of the steps other than Step 6 in Figure 5 as follows:

(i) the cell (re)selection when the UE joins the network in Step 1 in Figures 4 and 5,

(ii) UE gathers measurement reports and feeds them back to the serving cell in Step 2 in Figures 4 and 5,

(iii) a periodically measurement check in Step 3 in Figures 4 and 5,

(iv) CCS selection in Step 4(A) in Figure 4 and Step 5 in Figure 5,

(v) handover condition check in Step 5 in Figure 4 and Step 7 in Figure 5,

(vi) CTP starts transmitting data to UEs in Step 6 in Figure 4 and Step 8 in Figure 5, 
(vii) serving cell indicates all cells in CTP to cancel all current CTP connections in Step 7 in Figure 4 and Step 9 in Figure 5,

(viii) serving cell instructs UE to handover to future serving cell in Step 8 in Figure 4 and Step 10 in Figure 5,

(ix) UE detaches the network in Step 9 in Figure 4 and Step 11 in Figure 5.

Steps 1 and 2 in both algorithms are the ordinary information gathering procedures. Similarly, Step 3 performs a standard periodical measurement check in both algorithms. Step 4(A) in Figure 4 and Step 5 in Figure 5 share the same concept of CCS selection based on the target cells with highest RSRP in measurement set. However, the CCS selection in Step 5 in Figure 5 can be used dynamically based on different requirements (such as delay constraint) rather than RSRP. Step 5 in Figure 4 and Step 7 in Figure 5 function as the standard handover condition check within CTP. Steps 6 to 9 in Figure 4 and Steps 8 to 11 in Figure 5 are identically the same where both algorithms transmit data from CTP to UEs if the handover condition check (in Step 5 in Figure 4 and Step 7 in Figure 5) was not satisfied; otherwise serving cell sends out handover control messages in CTP for UE to be handed over to future serving cell. Lastly, UE detaches the network and repeats the cell (re)selection (Step 1 in Figure 4 and Figure 5) in both algorithms.

\section{Performance Metrics}

The system performances of CoMP Handover Algorithm and Limited CoMP Handover Algorithm are evaluated on the basis of RB Utilization, system throughput, and system delay. Detailed descriptions of each metric are provided below.

RB Utilization evaluates the proportion of total used RBs to total RBs in each cell and describes the current load state of the cell. A higher RB Utilization indicates a higher saturated state the cell becomes; therefore a cell reselection needs to be considered when more UEs are going to be handed over to this cell. On the other hand, when the cell is having a lower RB Utilization, this cell is capable of accommodating more incoming UEs. RB Utilization can be mathematically expressed as

$$
\mathrm{RB}_{\text {utilization }}=\frac{\sum_{t=1}^{T} \sum_{c=1}^{C} \mathrm{RB}_{\mathrm{used}_{c}}(t)}{\sum_{t=1}^{T} \sum_{c=1}^{C} \mathrm{RB}_{\max _{c}}(t)}
$$

where $C$ is the total number of cells, $T$ represents the total simulation time, $\mathrm{RB}_{\text {used }_{c}}(t)$ denotes the total resource block used of cell $c$ at time $t$, and $\mathrm{RB}_{\max _{c}}(t)$ denotes the total resource of cell $c$ at time $t$.

System throughput is defined as the total number of bits correctly received by all users and can be mathematically expressed as

$$
\text { system throughput }=\frac{1}{T} \sum_{t=1}^{T} \sum_{i=1}^{I} p_{\text {transmit }_{\_} i}(t) \quad c \forall \mathrm{CTP}_{i},
$$

where $I$ is the total number of UEs, $T$ represents the total simulation time, and $p_{\text {transmit }_{c} i}(t)$ denotes the number of transmitted bits of cell $c$ whichever earlier received by UE $i$ at time $t$. Cell $c$ belongs to CTP of UE $i$.

System delay gives the average of the total queuing delay of all packets in the buffers at the eNB in the system. This metric is defined as the average delay of Head-of-Line (HOL) packets of all UEs for the whole simulation time. System delay can be mathematically expressed as

$$
\text { system Delay }=\frac{1}{T} \sum_{t=1}^{T} \sum_{i=1}^{I} W_{c_{-} i}(t) \quad c \forall \mathrm{CTP}_{i},
$$

where $I$ is the total number of UEs, $T$ represents the total simulation time, and $W_{c \_i}(t)$ denotes the delay of the HOL packet of cell $c$ whichever earlier received by UE $i$ at time $t$. Cell $c$ belongs to CTP of UE $i$.

\section{Simulation Environment}

The performances of CoMP Handover Algorithm and Limited CoMP Handover Algorithm are evaluated and compared using a downlink LTE-A system based on [16, 17]. The simulation consists of 7 hexagonal cells of $5 \mathrm{MHz}$ bandwidth with $25 \mathrm{RBs}$ and $2 \mathrm{GHz}$ carrier frequency with 7 OFDM symbols over a slot of $0.5 \mathrm{~ms}$ duration. Users are uniformly distributed within the rectangle area as shown in Figure 6.

Each eNodeB is located at the center of each cell with 100 meter radius, and equal transmit power $(43.01 \mathrm{dBm}$ total eNodeB transmit power) is assumed on each RB. Each UE is constantly moving at $120 \mathrm{~km} / \mathrm{hr}$ speed. Each UE's direction is randomly initialled between 0 and $2 \pi$ and stays constant throughout the simulation. A reflect wraparound method is applied when each UE reaches the system boundary. The Cost-231 HATA model for an urban environment [18] is used to compute path loss. A Gaussian lognormal distribution with 0 mean and $8 \mathrm{~dB}$ standard deviation [19] is used for modelling shadow fading, and nonfrequency selective Rayleigh fading [20] is used to model the radio propagation channel. QPSK, 16QAM, and 64QAM are used as modulation and coding schemes. The Hybrid Automatic Repeat Request (HARQ) technique in [21] is adopted to recover wireless transmission errors. The maximum number of retransmissions is limited to 3. Round robin is selected as packet scheduling algorithm is to ensure the equal fairness for each UE in the system. A selected range of $\mathrm{HOM}$ as $1,2,3,4$, and $5 \mathrm{~dB}$ is used to optimize Limited CoMP Handover Algorithm. The sizes of CCS and CTP are set the same as the example provided in Figure 2, to 3 and 2 , respectively. The complete system parameters used in the simulation are listed in Table 1.

\section{Simulation Results}

The simulation results of Limited CoMP Handover Algorithm is presented and compared with CoMP Handover Algorithm in this section. Each performance metric is separately discussed in the following subsections. 


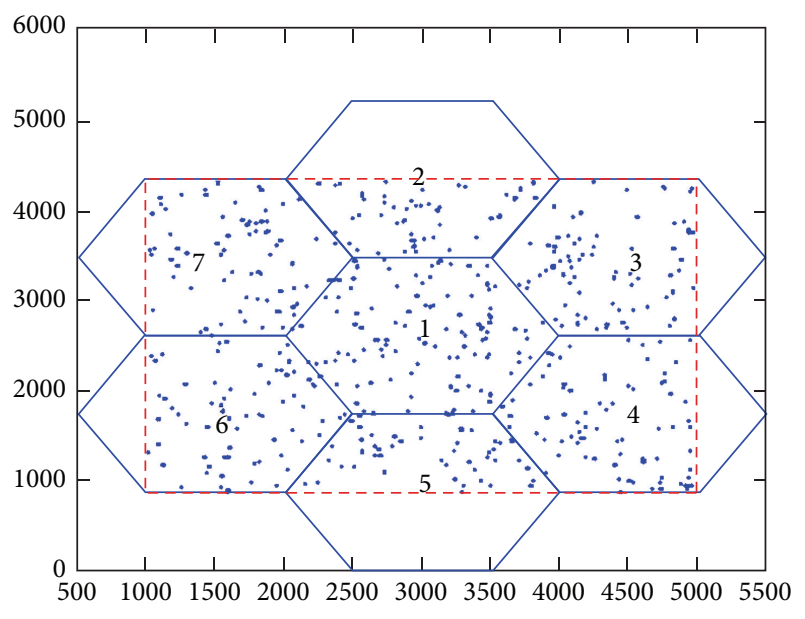

FIGURE 6: Simulation environment.

5.1. RB Utilization. Figure 7 illustrates the RB Utilization of CoMP and Limited CoMP Handover Algorithm in LTEA. The RB Utilization will increase when number of UEs increases in both algorithms because the more UEs are coming in the simulation, the more RBs have to be used for the users to transmit packets; therefore the RB Utilization will eventually reach $100 \%$ in the simulation when number of users increases in both algorithms. CoMP Handover Algorithm has higher RB Utilization over Limited CoMP Handover Algorithm in all UEs' scenarios. This is because CoMP Handover Algorithm has the fact of having two transmissions at all time, which requires at most twice as many RBs in the simulation compared to Limited CoMP Handover Algorithm.

In the case of 30 UEs in the simulation, CoMP Handover Algorithm already reaches $72.94 \%$ RB Utilization, whereas Limited CoMP Handover Algorithm reaches 57.18\%, 58.11\%, $62.11 \%, 63.90 \%$, and $65.18 \%$ RB Utilization at HOM equal to $1,2,3,4$, and 5, respectively. Furthermore, the saturated point where CoMP Handover Algorithm reaches a full 100\% RB Utilization state is at where 100 UEs are in the simulation while Limited CoMP Handover Algorithm reaches a full $100 \% \mathrm{RB}$ Utilization state at where $150 \mathrm{UE}$ are in the simulation. HOM equals 1 gives the lowest RB Utilization which overcomes other HOM values in Limited CoMP Handover Algorithm. The system becomes saturated after the number of UE is equal to 150 in both algorithms.

5.2. System Throughput. Figure 8 demonstrates the system throughput comparison of CoMP Handover Algorithm and Limited CoMP Handover Algorithm in LTE-A. The overall trend of system throughput increases in both algorithms when the number of UEs increases because the more UEs coming in the simulation, the more successfully transmit packets from all UEs in the system. According to (5), the higher the number of transmitted bits in the system is, the higher the system throughput it becomes. CoMP Handover Algorithm provides better system throughput as $27.28 \mathrm{Mbps}$ and 42.02 Mbps over Limited CoMP Handover Algorithm
TABLE 1: Simulation parameters.

\begin{tabular}{ll}
\hline Parameters & Values \\
\hline Cellular layout & $\begin{array}{l}\text { Hexagonal grid, wrap around } \\
\text { (reflect), 7 cells }\end{array}$ \\
Radius & $100 \mathrm{~m}$ \\
Carrier frequency & $2 \mathrm{GHz}$ \\
Bandwidth & $5 \mathrm{MHz}$ \\
SubCarrier spacing & $15 \mathrm{kHz}$ \\
Slot duration & $0.5 \mathrm{~ms}$ \\
Number of OFDM symbols/slot & 7 \\
Number of RBs & 25 \\
Number of subcarriers per RB & 12 \\
Path loss & Cost 231 Hata model \\
Shadow fading & Gaussian log normal \\
distribution model \\
Multipath & Nonfrequency selective \\
Modulation and coding scheme & QPayleigh fading model \\
Packet scheduler & Round robin \\
Scheduling time (TTI) & $1 \mathrm{~ms}$ \\
Data traffic & $1 \mathrm{Mbps}$ constant stream \\
Number of UE & $30,50,100,150,200,250$, and \\
UE's position & 300 \\
UE's direction & Fixed uniform distributed \\
UE's velocity & Randomly choose from [0, \\
Simulation time & $2 \pi]$, constant at all time \\
RSRP measurement period & $120 \mathrm{~km} / \mathrm{hr}$ \\
Handover margin & $5000 \mathrm{~ms}$ \\
Time to trigger (TTT) & $10 \mathrm{~ms}$ \\
Size of CCS & $\{1,2,3,4,5\}$ dB \\
\hline
\end{tabular}

regardless of the HOM value in the scenario of 30 and 50 UEs, respectively. This is because when the number of UEs is low, the RB Utilization of CoMP Handover Algorithm has yet reached the saturated state (Figure 7); hence the UEs in CoMP Handover Algorithm can fully utilize all the available RBs in the network, which directly improve the system throughput in the simulation. On the other hand, Limited CoMP Handover Algorithm restricts the number of data connections for each UE at any time in the system based on their channel condition. Even when there are still plenty of available RBs remaining in the system, Limited CoMP Handover Algorithm only supports one single data connection at a time for UEs in a fair/good channel condition, which limits the number of transmitted bits in the system, directly limiting the system throughput in the system. Therefore CoMP Handover Algorithm can reach a higher system throughput over Limited CoMP Handover Algorithm when in a low saturated system. 


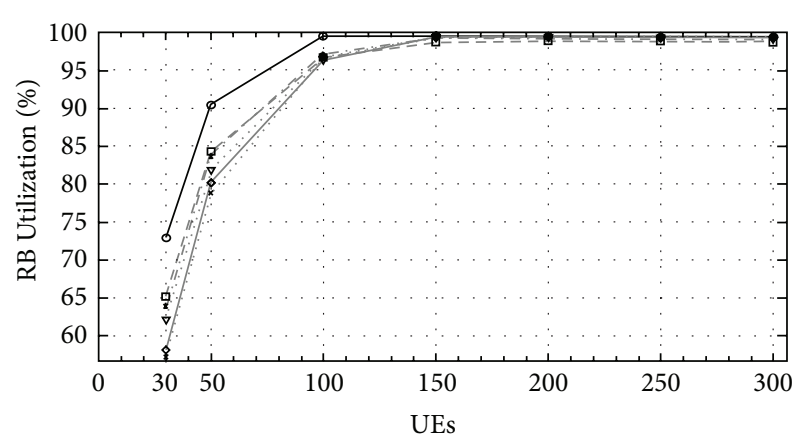

- CoMP Handover Algorithm
$\times \quad$ Limited CoMP Handover Algorithm, HOM $=1$
$\diamond \quad$ Limited CoMP Handover Algorithm, HOM $=2$
$\nabla \cdot$ Limited CoMP Handover Algorithm, HOM $=3$
$-* \quad$ Limited CoMP Handover Algorithm, HOM $=4$
$-\boxminus-$ Limited CoMP Handover Algorithm, HOM $=5$

FIgURE 7: RB Utilization of CoMP Handover Algorithm and Limited CoMP Handover Algorithm in LTE-A.
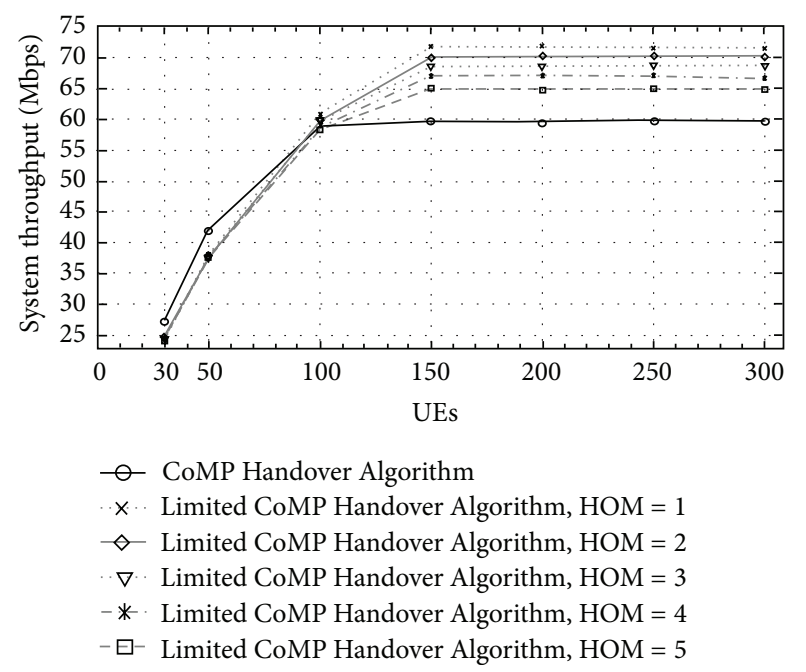

FIgure 8: System Throughput of CoMP Handover Algorithm and Limited CoMP Handover Algorithm in LTE-A.

However when the system is at 150 UEs, Limited CoMP Handover Algorithm outperforms CoMP Handover Algorithm with the system throughput improvement of $19.91 \%$, $17.09 \%, 14.64 \%, 12.02 \%$, and $8.65 \%$ when HOM equals 1,2 , 3, 4, and 5, respectively. Limited CoMP Handover Algorithm takes the advantage from supporting single data connection for cell-center UEs in the system. A cell-center UE in CoMP Handover Algorithm will allocate two data transmissions at any time, while in Limited CoMP Handover Algorithm, a cell-center UE can only allocate one single data transmission. This restriction frees the available RBs of the second data connection from the cell-center UE, and the freed available $\mathrm{RBs}$ can be further used for other new incoming UE which improves more transmit packets in the system and therefore enhances the system throughput. When the system becomes saturated, the system throughput stops increasing due to

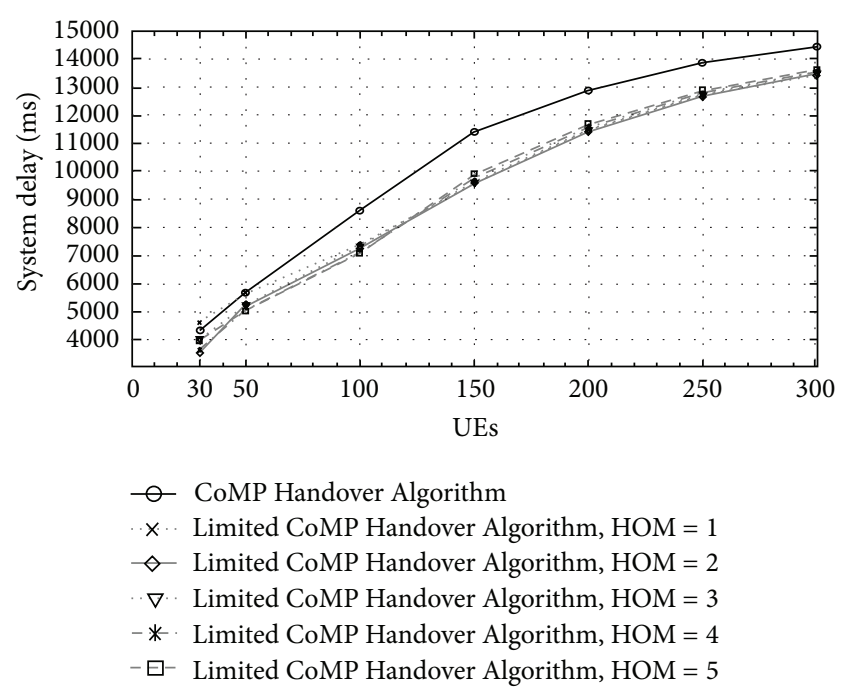

FIGURE 9: System Delay of CoMP Handover Algorithm and Limited CoMP Handover Algorithm in LTE-A.

insufficient radio resources (RBs) to be allocated to the UEs in the system. It is the fact that the system throughput of CoMP Handover Algorithm stops increasing and stays fixed around $60 \mathrm{Mbps}$ at 150, 200, 250, and 300 UEs' scenarios. When the system becomes saturated in 150, 200, 250, and 300 UEs' scenarios, the system throughput of Limited CoMP Handover Algorithm also stops increasing and stays fixed at $72 \mathrm{Mbps}$, $70 \mathrm{Mbps}$, $68 \mathrm{Mbps}$, $67 \mathrm{Mbps}$, and $65 \mathrm{Mbps}$ where HOM equals $1,2,3,4$, and 5 , respectively. HOM equal to 5 in Limited CoMP Handover Algorithm has the lowest system throughput due to the fact of having the largest HOM value which delays the handover triggering timing. A too late handover will affect the channel condition of the UE and also a too late handover would be more likely leading to a radio link failure situation where the packets of the UE cannot be correctly received which reduces the system throughput.

5.3. System Delay. Figure 9 shows the system delay comparison of CoMP Handover Algorithm and limited CoMP Handover Algorithm in LTE-A. The overall trend of system delay increases in both algorithms when the number of UEs increases because the more UEs are coming in the simulation, the more condensed the system becomes where all the packets need to be buffered longer in the queue to get transmitted to all the UEs, which leads to higher system delay. CoMP Handover Algorithm has lower system delay as $4341.33 \mathrm{~ms}$ than the delay of Limited CoMP Handover Algorithm when HOM equals to 1 as $4597.8 \mathrm{~ms}$ is because the total capacity of the cells in the simulation is not yet fully utilized, which means the total available RBs in the cells can be fully used to transmit packets for each UE; thus the time for packets to be waited and queued would not last long in the buffer; therefore CoMP Handover Algorithm reaches a lower system delay result. CoMP Handover Algorithm has higher system delay as $5691.81 \mathrm{~ms}, 8615.15 \mathrm{~ms}, 11416.7 \mathrm{~ms}, 12874.8 \mathrm{~ms}$, $13904.8 \mathrm{~ms}$, and $14455 \mathrm{~ms}$ over Limited CoMP Handover 
Algorithm regardless of the $\mathrm{HOM}$ value in the scenario of 50 , $100,150,200,250$, and 300 UEs, respectively. This is because every UE's packet in either serving cell and/or target cell in CTP has to be buffered and queued to be transmitted in the system; while CoMP Handover Algorithm fully uses two data connections for each UE at each time instant in the simulation, the packets that belong to the UE need to be buffered in both of the serving cell and the target cell in CTP. This behaviour increases the loading and the queuing delay of both of the serving cell and the target cell in CTP which leads to higher system delay. In Limited CoMP Handover Algorithm, when a cell-edge UE only can be offered a single data connection, this UE will not have the chance to have the second data connection from the target cell in CTP; thus there will be no packet to be buffered and queued in the target cell in CTP for this particular UE. Therefore this UE will not increase the loading and the queuing delay of the target cell in CTP which leads to a lower system delay result.

\section{Conclusions}

A new handover algorithm named Limited CoMP Handover Algorithm is proposed in this paper and its impact on a number of optimized handover parameters under the downlink LTE-A system is evaluated. The performance of the proposed algorithm is compared with the open literature handover algorithm under different number of UEs' scenarios. It is shown via simulation that the proposed handover algorithm can improve the system throughput compared to CoMP Handover Algorithm in a saturated system when UE is equal to $150,200,250$, and 300 . The proposed handover algorithm is able to maintain a lower system delay when compared with CoMP Handover Algorithm. Moreover, the system throughput and system delay of proposed handover algorithm can be further improved by optimizing the HOM variable. Evaluating the performance of the proposed handover algorithm under different wireless scenarios and taking QoS requirements of multimedia services under consideration will be the focus of the future studies.

\section{References}

[1] ITU-R M.2134, "Requirements related to technical performance for IMT-Advanced radio interface(s)," 2008.

[2] M. Kottkamp, "LTE-Advanced Technology Introduction White Paper," Rohde \& Schwarz GmbH \& Co. KG, 2010.

[3] H. Holma and A. Toskala, LTE for UMTS-Evolution to LTEAdvanced, 2011.

[4] A. Omri, R. Bouallegue, R. Hamila, and M. Hasna, "Channel estimation for LTE uplink system by perceptron neural network," International Journal of Wireless \& Mobile Networks, vol. 2, pp. 155-165, 2010.

[5] D. Singhal, M. Kunapareddy, V. Chetlapalli, V. B. James, and N. Akhtar, "LTE-advanced: handover interruption time analysis for IMT-A evaluation," in Proceedings of the International Conference on Signal Processing, Communication, Computing and Networking Technologies (ICSCCN '11), pp. 81-85, 2011.

[6] A. Ghosh, R. Ratasuk, B. Mondal, N. Mangalvedhe, and T. Thomas, "LTE-advanced: next-generation wireless broadband technology," IEEE Wireless Communications, vol. 17, no. 3, pp. $10-22,2010$.

[7] S. Redana and B. Raaf, "LTE-A relaying tutorial," in Proceedings of the IEEE Wireless Communications and Networking Conference (WCNC '11), Cancun, Mexico, 2011.

[8] 3GPP TR36.912 V10.0.0, "3rd Generation Partnership Project; Technical Specification Group Radio Access Network; Feasibility study for Further Advancements for E-UTRA (LTEAdvanced) (Release 10)," 2011.

[9] R. Y. Kim, I. Jung, X. Yang, and C. C. Chou, "Advanced handover schemes in IMT-advanced systems," IEEE Communications Magazine, vol. 48, no. 8, pp. 78-85, 2010.

[10] X. Xiaodong, C. Xin, and L. Jingya, "Handover mechanism in coordinated multi-point transmission/reception system," ZTE Communications, no. 1, 2010.

[11] C. C. Lin, K. Sandrasegaran, and S. Reeves, "Handover algorithm with joint processing in LTE-advanced," in Proceedings of the 9th International Conference on Electrical Engineering/Electronics, Computer, Telecommunications and Information Technology (ECTI-CON '12), pp. 1-4, 2012.

[12] N. Zheng and J. Wigard, "On the performance of integrator handover algorithm in LTE networks," in Proceedings of the 68th Semi-Annual IEEE Vehicular Technology Conference (VTC '08), pp. 1-5, Calgary, Canada, September 2008.

[13] M. Anas, F. D. Calabrese, P. E. Östling, K. I. Pedersen, and P. E. Mogensen, "Performance analysis of handover measurements and layer 3 filtering for UTRAN LTE," in Proceedings of the18th Annual IEEE International Symposium on Personal, Indoor and Mobile Radio Communications (PIMRC '07), pp. 1-5, September 2007.

[14] J. Puttonen, J. Kurjenniemi, and O. Alanen, "Radio problem detection assisted rescue handover for LTE," in Proceedings of the IEEE 21st International Symposium on Personal Indoor and Mobile Radio Communications (PIMRC '10), pp. 1752-1757, September 2010.

[15] T. Jansen, I. Balan, J. Turk, I. Moerman, and T. Kürner, "Handover parameter optimization in LTE self-organizing networks," in Proceedings of the IEEE 72nd Vehicular Technology Conference Fall (VTC '10), pp. 1-5, September 2010.

[16] C. C. Lin, K. Sandrasegaran, H. A. M. Ramli et al., "Optimization of handover algorithms in 3GPP long term evolution system," in Proceedings of the 4th International Conference on Modeling, Simulation and Applied Optimization (ICMSAO '11), Kuala Lumpur, Malaysia, April 2011.

[17] H. A. M. Ramli, K. Sandrasegaran, R. Basukala, and L. Wu, "Modeling and simulation of packet scheduling in the downlink long term evolution system," in Proceedings of the 15th AsiaPacific Conference on Communications (APCC '09), pp. 68-71, Shanghai, China, October 2009.

[18] H. H. A. Toskala, WCDMA for UMTS: Radio Access for Third Generation Mobile Communications, John Wiley \& Sons, Nokia, Finland, 3rd edition, 2004.

[19] M. Gudmundson, "Correlation model for shadow fading in mobile radio systems," Electronics Letters, vol. 27, no. 23, pp. 2145-22146, 1991.

[20] C. Komninakis, "A Fast and accurate rayleigh fading simulator," in Proceedings of the IEEE Global Telecommunications Conference (GLOBECOM '03), vol. 6, pp. 3306-3310, December 2003.

[21] J. C. Ikuno, M. Wrulich, and M. Rupp, "Performance and modeling of LTE H-ARQ," in Proceedings of the International ITG Workshop on Smart Antennas (WSA '09), Fraunhofer Heinrich-Hertz-Institut, Berlin, Germany, 2009. 

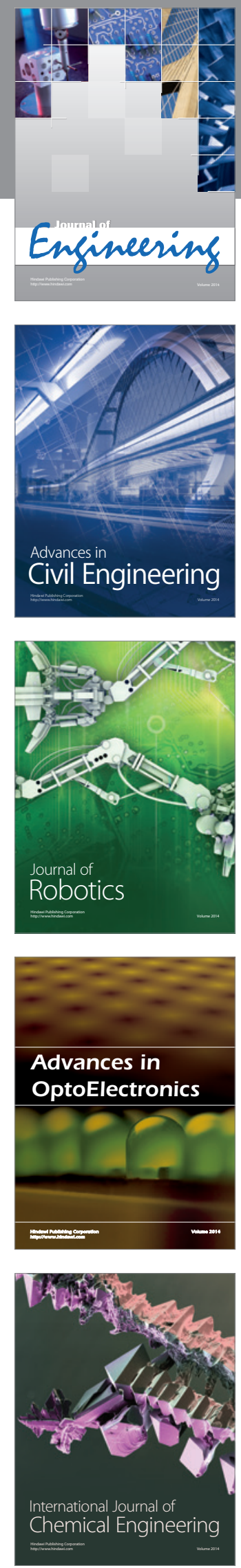

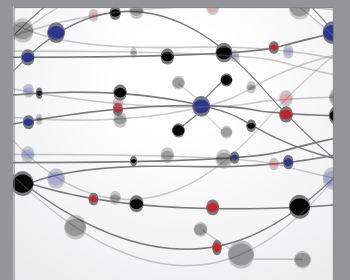

The Scientific World Journal
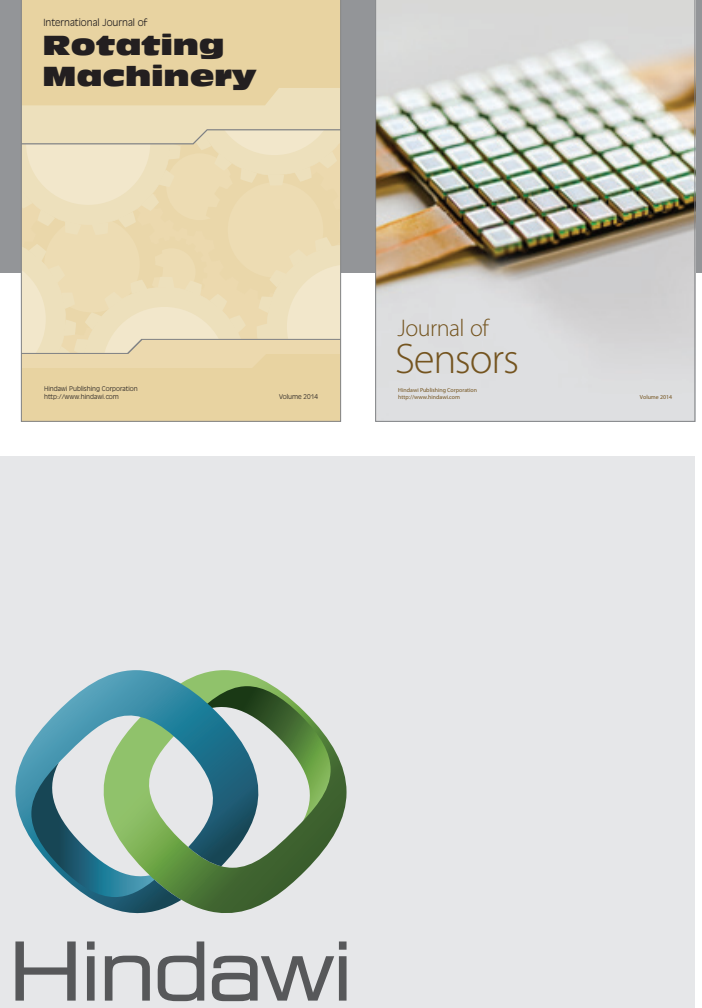

Submit your manuscripts at http://www.hindawi.com
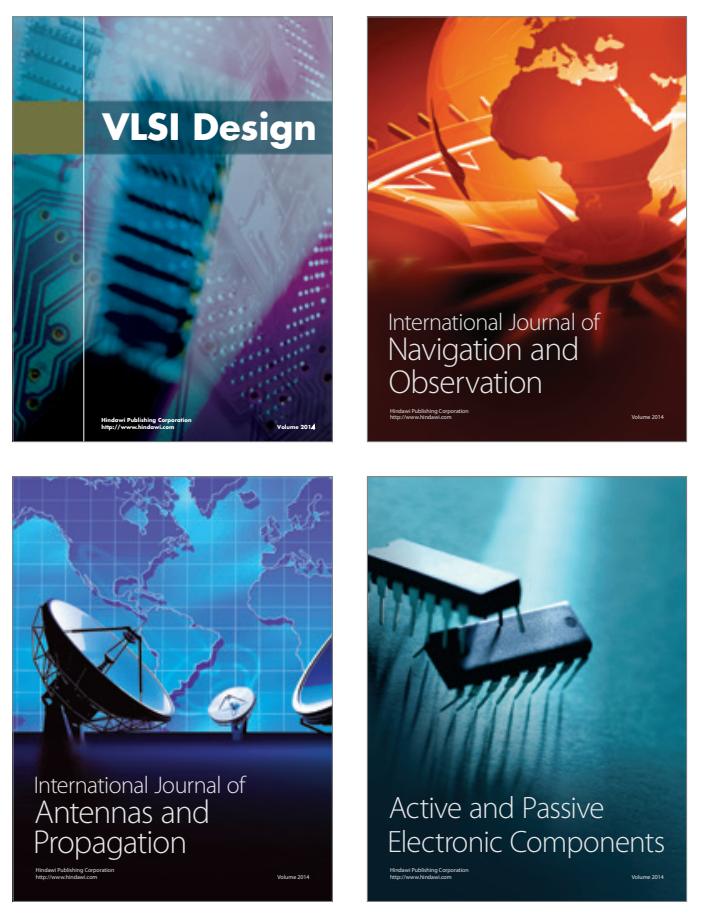
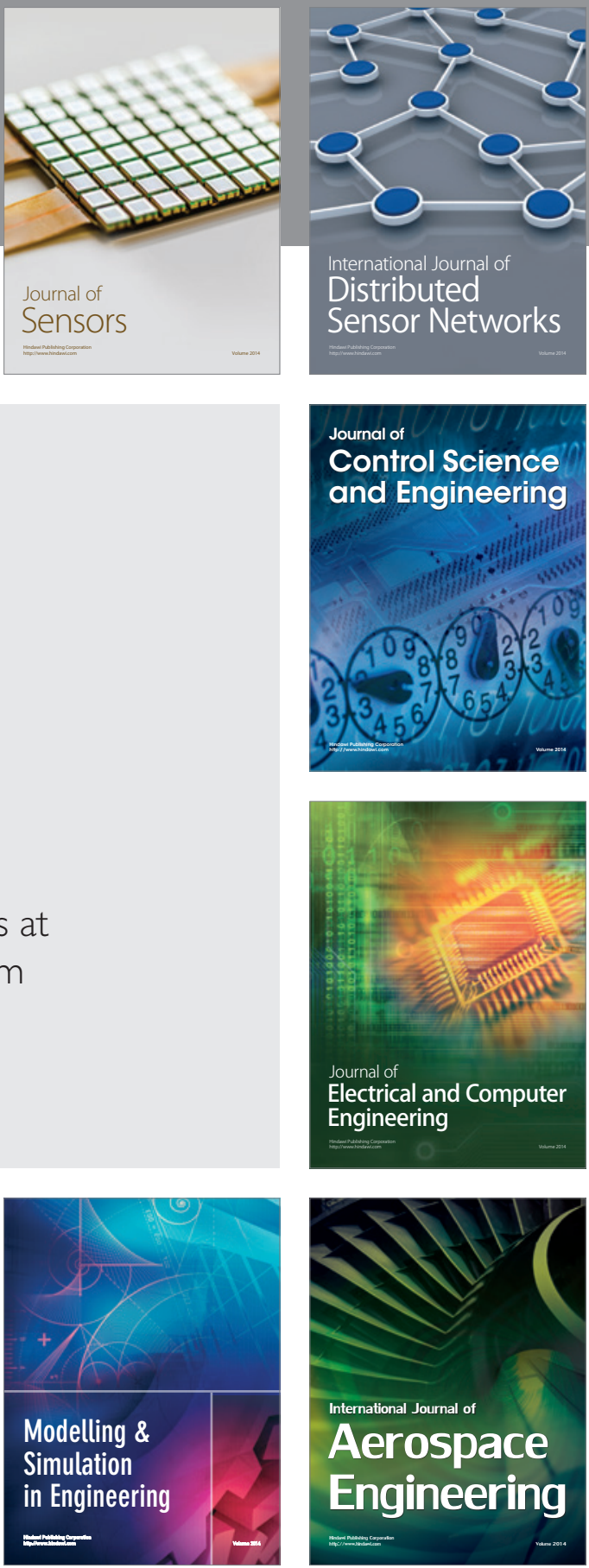

Journal of

Control Science

and Engineering
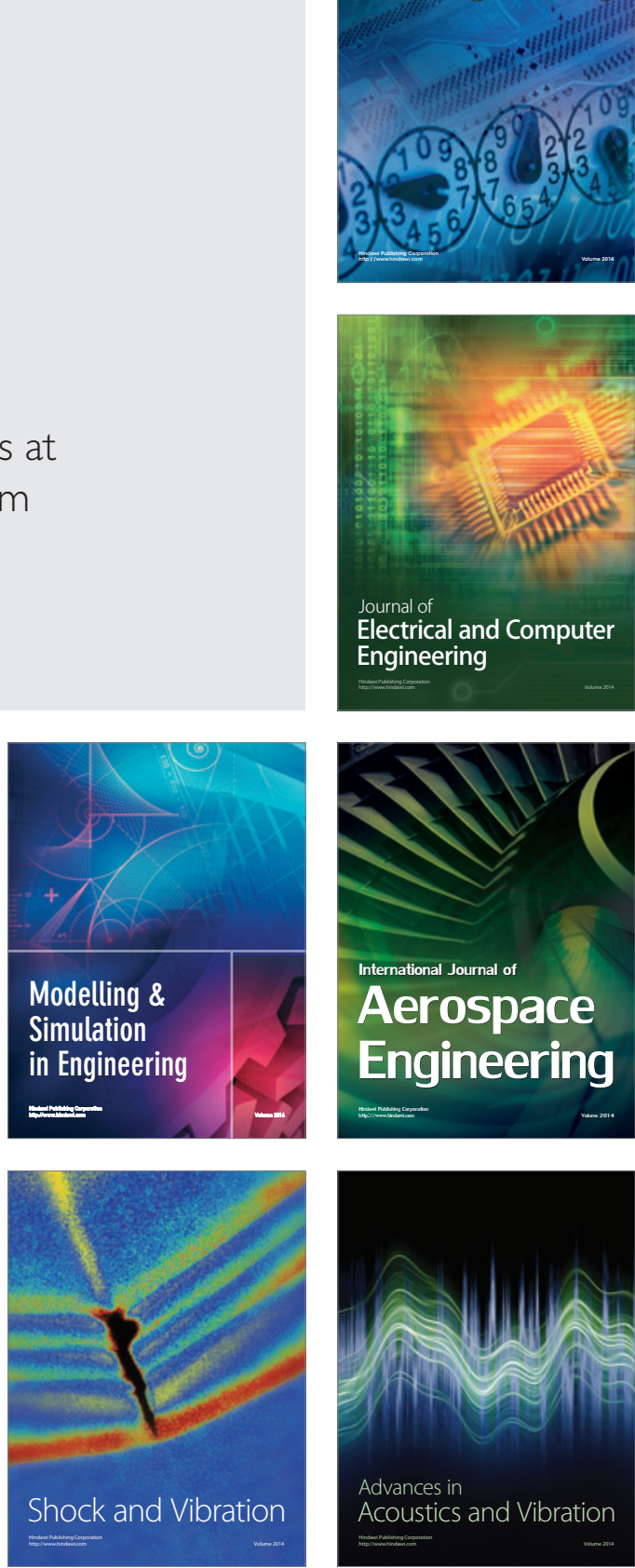